\title{
ANALYSIS AND SIMULATION OF HADRONIC SHOWERS IN A URANIUM GAS-SAMPLING CALORIMETER
}

\author{
L3 Collaboration \\ CERN, Geneva, Switzerland
}

\author{
A. AREFIEV 1), T. AZEMOON ${ }^{2)}$, H.S. CHEN ${ }^{3)}$, G.M. CHEN ${ }^{3)}$, Yu. GALAKTIONOV 1), \\ Yu. KAMYSHKOV ${ }^{1)}$, A. KUNIN ${ }^{1)}$, P. LE COULTRE ${ }^{4)}$, J. LETTRY ${ }^{4)}$, J.B. MILLS ${ }^{2)}$, \\ V. PLYASKIN ${ }^{1)}$, V. SHOUTKO ${ }^{1)}$, E. SHUMILOV ${ }^{1)}$, B. SPIESS ${ }^{4)}$ and J. ULBRICHT ${ }^{\text {) }}$ \\ 1) Institute for Theoretical and Experimental Physics, Moscow, USSR \\ 2) University of Michigan, Ann Arbor, MI, USA \\ 3) Institute of High-Energy Physics, Beijing, P.R. China \\ 4) Eidgenossische Technische Hochschule, Zürich, Switzerland \\ 5) Paul Scherrer Institute, Würenlingen, Switzerland
}

Received 8 September 1989

The results of a simulation of the response of the $\mathrm{L} 3$ detector to hadrons is presented. The simulation is made on the basis of data obtained in the beam with the setup consisting of a uranium gas-sampling calorimeter with a bismuth germanium oxide calorimeter in front of it.

\section{Introduction}

The uranium gas-sampling calorimeter of the L3 experiment, together with the bismuth germanium oxide (BGO) calorimeter situated in front of it, is used to measure the hadronic energy flow. The measurement of hadronic energy in a combined system of essentially different detectors, i.e. a homogeneous BGO calorimeter and a sampling hadron calorimeter, requires a knowledge of the development of hadronic showers in these detectors. In view of that, we have performed a study of hadronic showers in a uranium gas-sampling calorimeter using the data taken at different energies in a CERN Super Proton Synchrotron (SPS) beam with a test setup which imitates the L3 detector structure [1] *.

In the following, the results of the analysis of these data are used to developed a model for the simulation of the hadron calorimeter response.

\section{Test setup}

The setup (fig. 1) consists of four modules of the L3 barrel hadron calorimeter (denoted by HC from now

- The details of the beam test will be published elsewhere. on) [1] put on a support installed behind the matrix consisting of $10 \times 10$ BGO crystals [2,3]. Each of the 24-cm long crystals has an area of $2.5 \times 2.5 \mathrm{~cm}^{2}$ at the front end and $3.5 \times 3.5 \mathrm{~cm}^{2}$ at the back end. The matrix is situated so that the beam line coincides with the axis of the central crystal. The BGO calorimeter (denoted by BGO alone from now on) has a thickness of 0.93 nuclear absorption lengths $(\lambda) * *$.

The HC modules consisi of 5-mm thick uranium absorber interleaved with proportional chambers [4] filled with an $\mathrm{Ar}-\mathrm{CO}_{2}$ mixture. The thickness of each layer is $0.058 \lambda$.

The signal wires of adjacent chambers are perpendicular to each other. The wires of the chambers corresponding to the same signal-wire orientation are grouped together in a readout cell pattern shown in fig. 2 . The number of chamber planes in a cell varies from 1 in the front to 4 in the rear part of the module. The typical size of a cell in the transverse direction is about $50 \mathrm{~mm}$.

To make the test setup as close as possible to that of the real L3 detector, the $0.21 \lambda$ thick steel pieces of the inner support ring of the barrel $\mathrm{HC}$ are mounted onto the side of the nodules facing the BGO.

\footnotetext{
* The nuclear absorption length depends on the kind of particle and its energy. The figures quoted correspond to 20-GeV pions.
} 


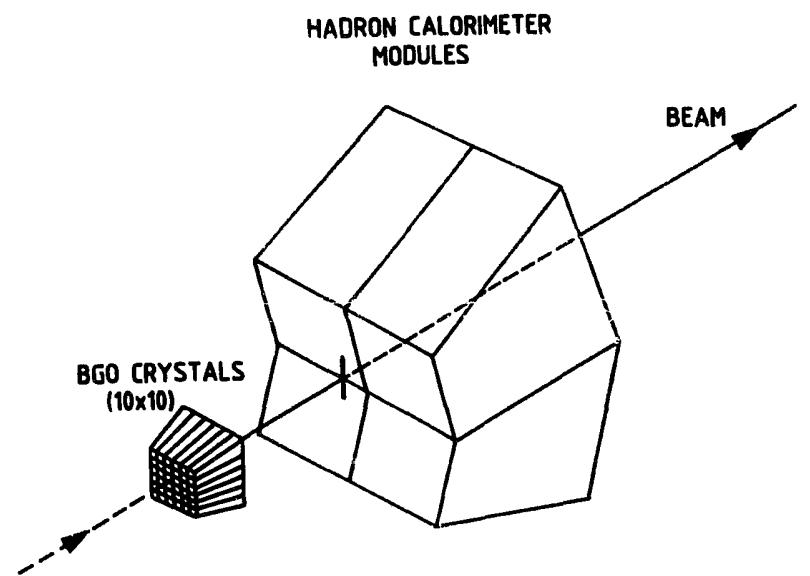

Fig. 1. Experimental setup.

Owing to the movable support the test setup can be put into different positions with respect to the beam, thus varying the angles of incidence.

The data were taken at different energies ranging from 4 to $20 \mathrm{GeV}$.

\section{Hadronic shower characteristics}

We begin the analysis with events where pions traverse the BGO without nuclear interaction, i.e. those giving a minimum ionizing particle signal in the BGO. In the following all signals are measured in units of minimum ionizing particle signal (mip), which we define as an average signal from an $12-\mathrm{GeV}$ muon traversing one HC chamber. The energy lost by the relativistic particle in the BGO is about $270 \mathrm{MeV}$.

The HC response to 20-GeV pions is shown in fig. 3. From the two distributions shown in this figure one can see that the HC response is almost entirely made up of the signals with large (>6 mip) amplitudes. The measured mean number of these amplitudes is close to 25 (fig. 4) per event and is considerably less than the measured total number of signals (about 44) in 2 hadronic shower (fig. 5).

In fig. 6 the spectra of the amplitudes of the signals produced by $20-\mathrm{GeV}$ pion showers are shown. One can see that the spectra have a two-component structure with small ( $<2 \mathrm{mip}$ ) and large amplitudes differently distributed in the transverse direction. The large amplitudes are narrowly distributed around the initial direction of the particle (fig. 7), whereas the transverse distribution of the small amplitudes is essentially different and is considerably wider (fig. 8). As for the longitudinal distributions of different signals, they are similar for all signals contributing to the $\mathrm{HC}$ response (fig. 9).
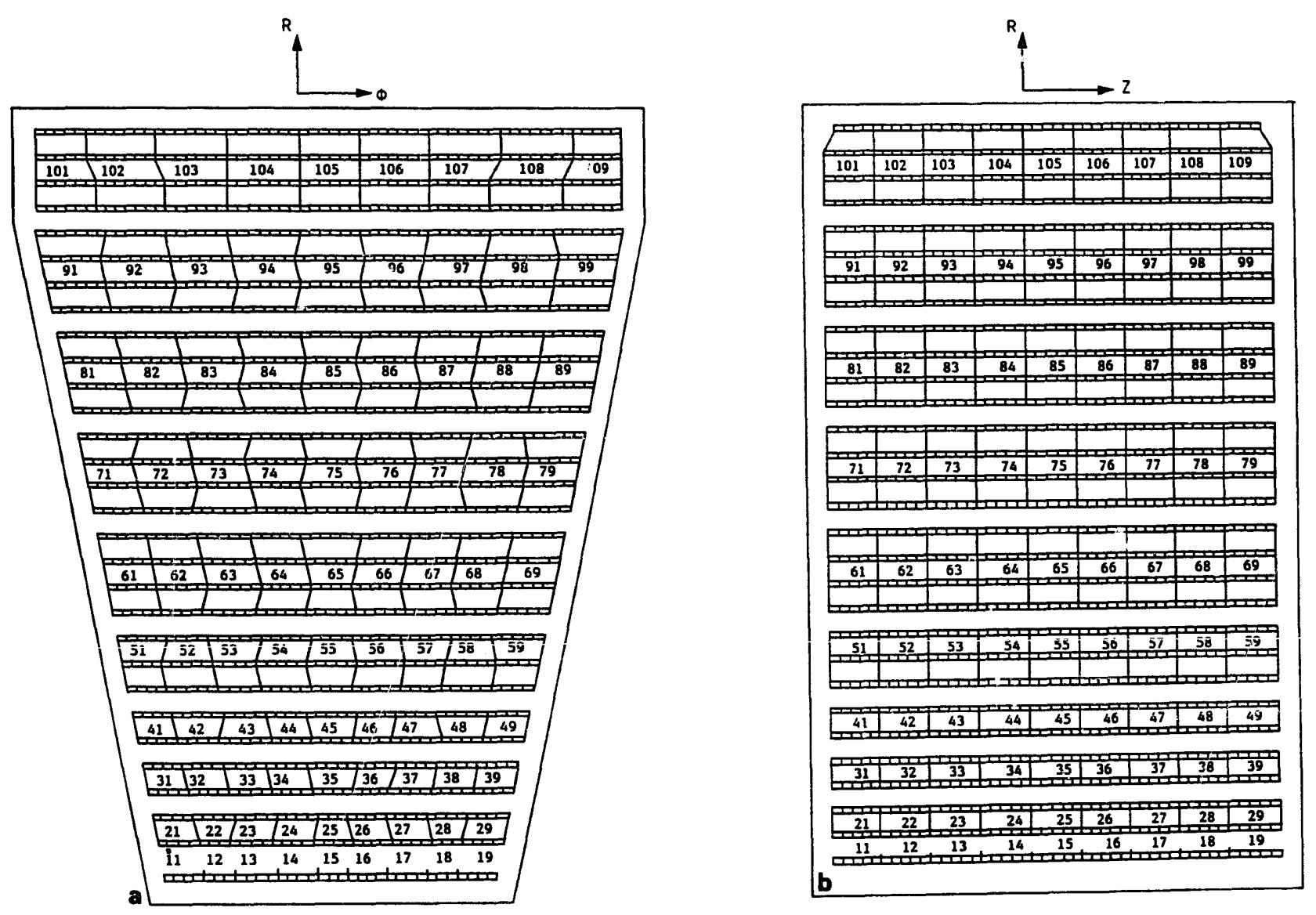

Fig. 2. Module readout pattern: (a) $\theta$ projection; (b) $\phi$ projection. 

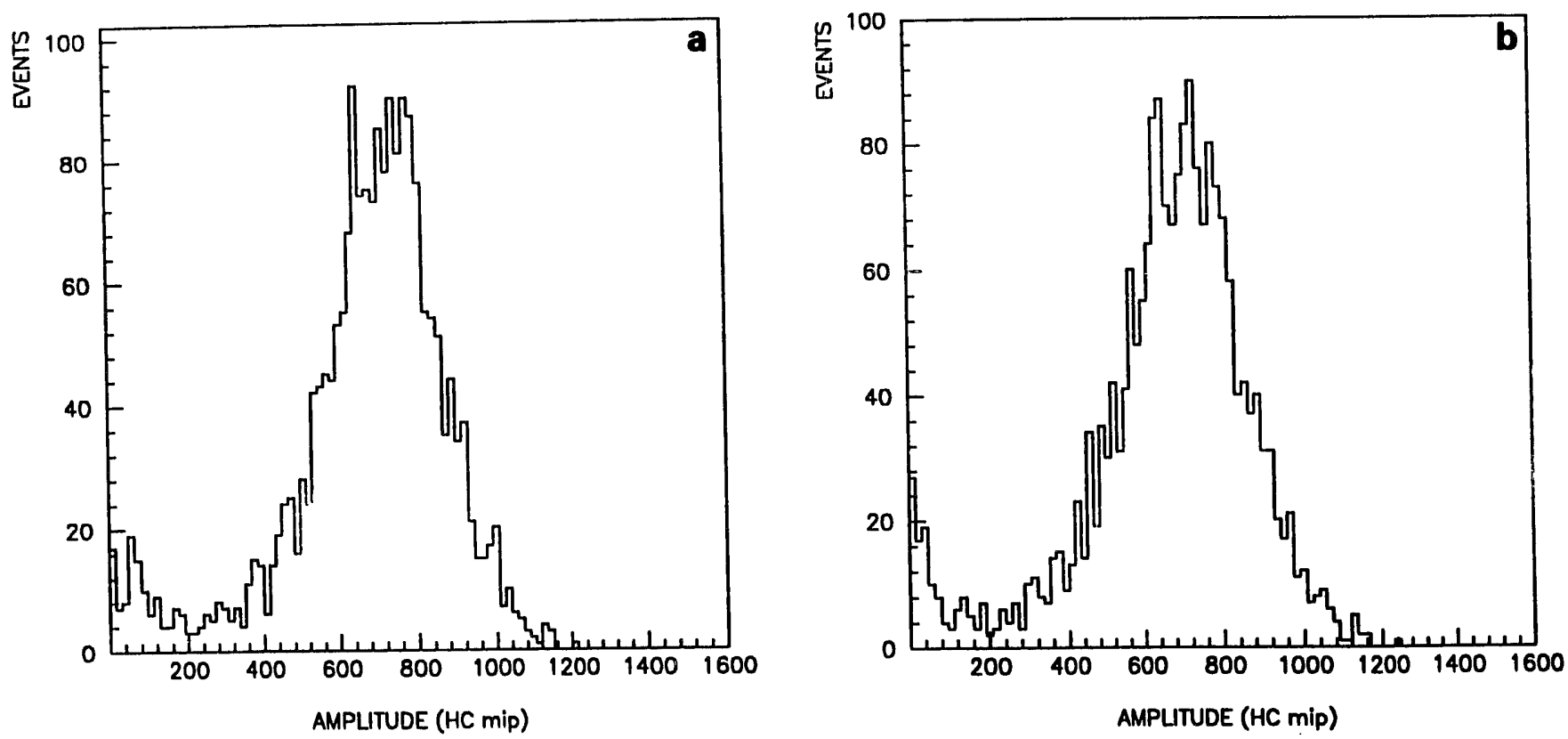

Fig. 3. The response of the HC to 20-GeV pions noninteracting in the BGO: (a) sum of all signals; (b) sum of signals with amplitude $>6$ mip.

The longitudinal distribution shown in fig. 9 corresponds to the hadronic shower averaged over different interaction points in the HC. The actual longitudinal shape of the individual shower is obtained when the starting point of the shower is fixed. In fig. 10 the longitudinal distribution of showers originating in the third and fourth layers of the HC is shown. This has a large peak in the beginning and a long tail.

The distribution shown in fig. 3 has an asymmetry arising from noncontainment of showers originating in

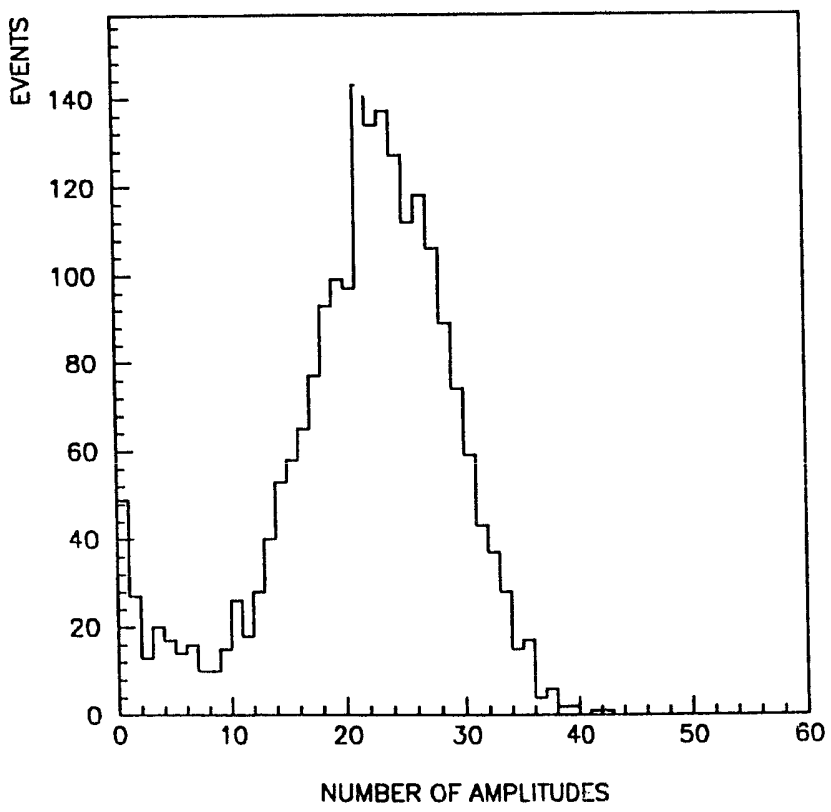

Fig. 4. Number of amplitudes $>6 \mathrm{mip}$ for $20-\mathrm{GeV}$ pions noninteracting in the BGO. the rear part of the HC. Selecting events with showers starting in the front end of the HC one obtains the distribution corresponding to practically full containment (fig. 11). This is symmetric and has a rms of $18 \%$.

The difference in transverse distributions of signals of different amplitudes explains the change of the amplitude spectra from cell to cell (fig. 6). All spectra can be fitted to two exponential functions. The slope of the exponential function describing small amplitudes is the

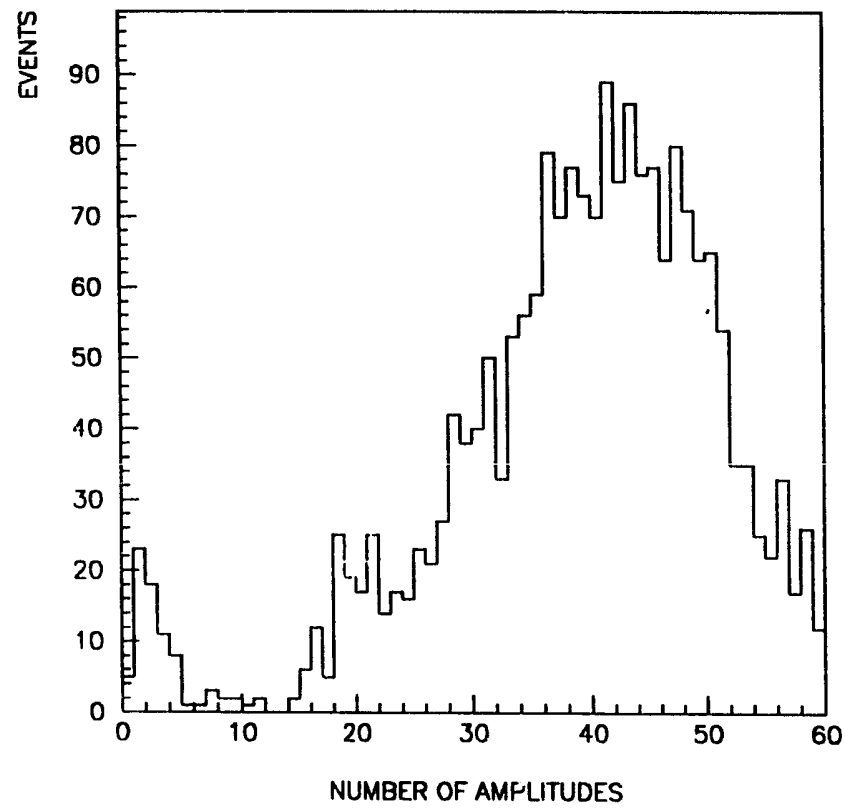

Fig. 5. Number of amplitudes $>1 \mathrm{mip}$ for $20-\mathrm{GeV}$ pions noninteracting in the BGO. 

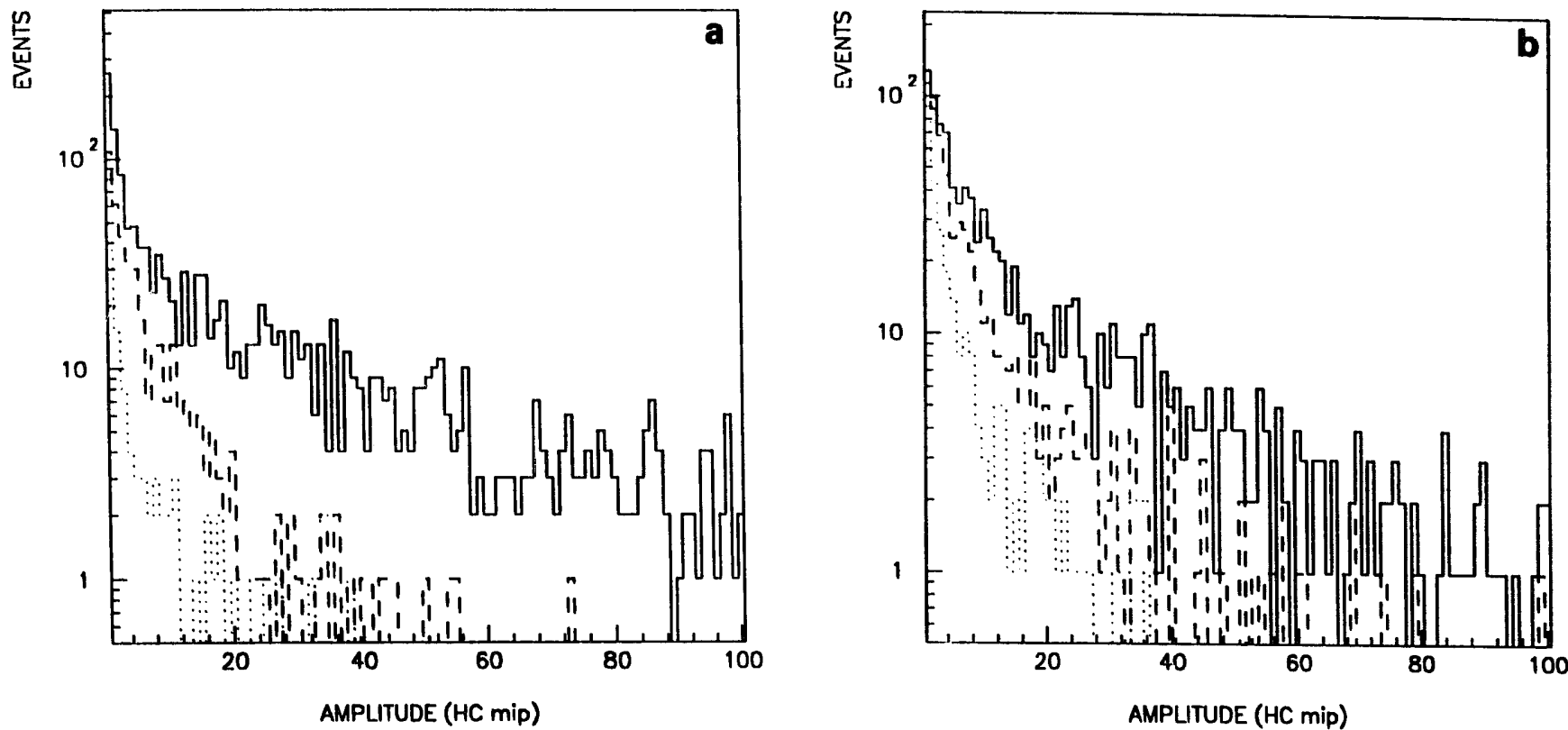

Fig. 6. Measured amplitude spectra in different cells. Solid line: cell number 5 (central); dashed line: cell number 3 (intermediate); dotted line: cell number 1 (peripheral); (a) layer number 3 (two chambers); (b) layer number 8 (four chambers).

same for all readout cells and is equal to $1.6 \mathrm{mip}$. On the contrary, the observed slope of the large amplitude exponential function is decreasing with the density of the shower. This behaviour can be explained by the coalescing of large signals caused by the readout-cell structure. When several signals occur in the same cell the amplitude spectrum is no longer only determined by the spectrum of signals proper, but also by the number of coalescing signals. Apart from that, because of

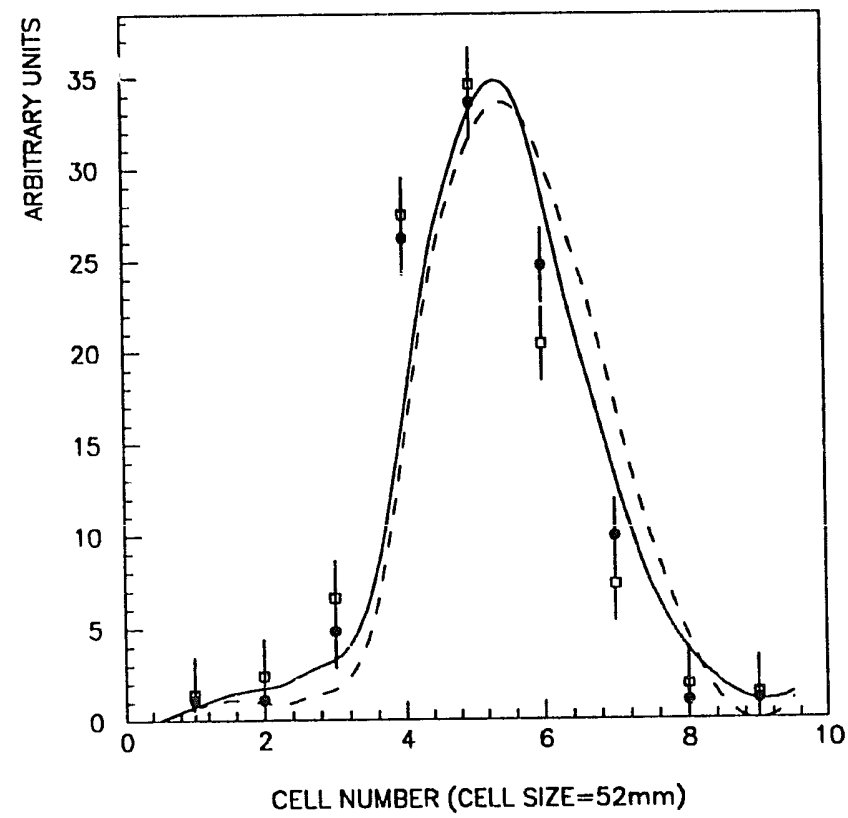

Fig. 7. Transverse distribution of large amplitudes obtained from the two-exponential fit of spectra in different cells. Open box: layer number 3; full point: layer number 8 . coalescence the detected number of amplitudes (figs. 4 and 5) is smaller than the actual one.

The data taken at different energies suggest that the spectra of amplitudes have the same two-component structure at all energies; the transverse distribution of the shower is almost energy-independent, whereas the longitudinal distribution depends logarithmically on the incident energy. From the data corresponding to differ-

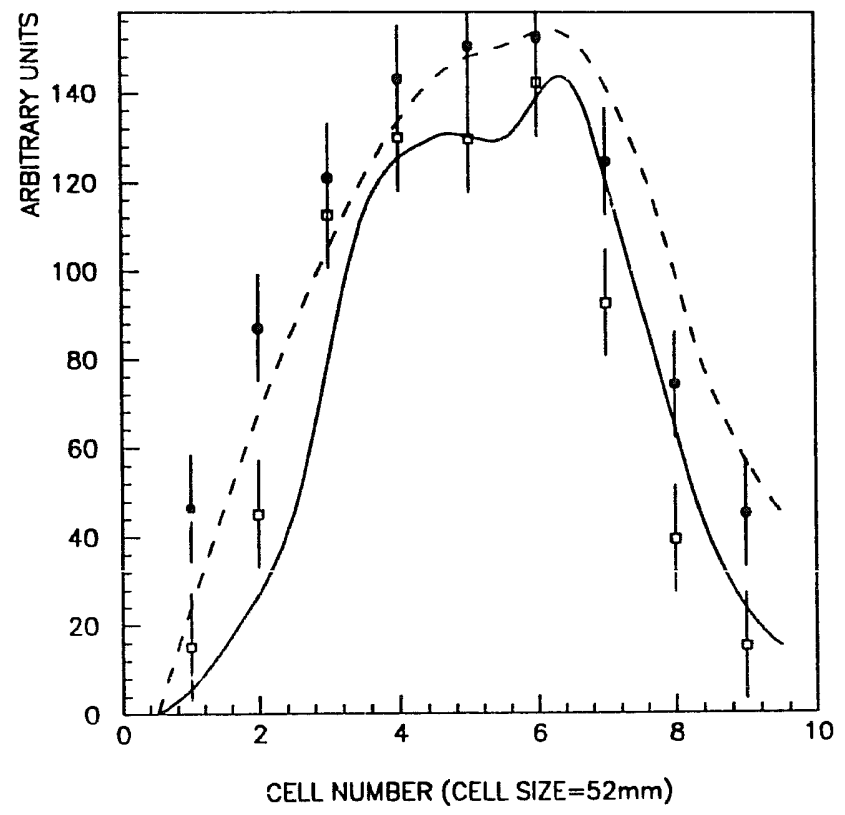

Fig. 8. Transverse distribution of small amplitudes obtained from the two-exponential fit of spectra in different cells. An open box represents layer number 3 ; a full point represents layer number 8 . 


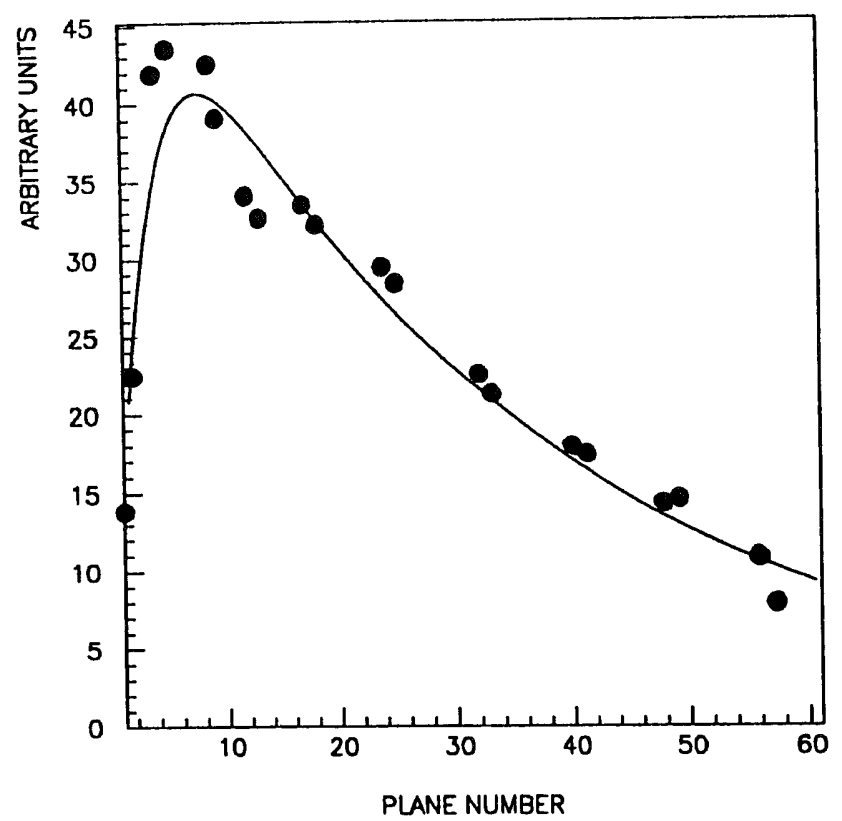

Fig. 9. Longitudinal distribution of hadronic showers in the HC. The curve is a result of the fit of data points to the function $\exp (-A x)[1-\exp (-B x)]$, where $A=0.33, B=0.33$. The $x$ is in planes of the HC. The thickness of one plane is $0.058 \lambda$.

ent angles we obtained a simple $\cos \theta$ dependence of the response of the $\mathrm{HC}$ on the angle of incidence of pions.

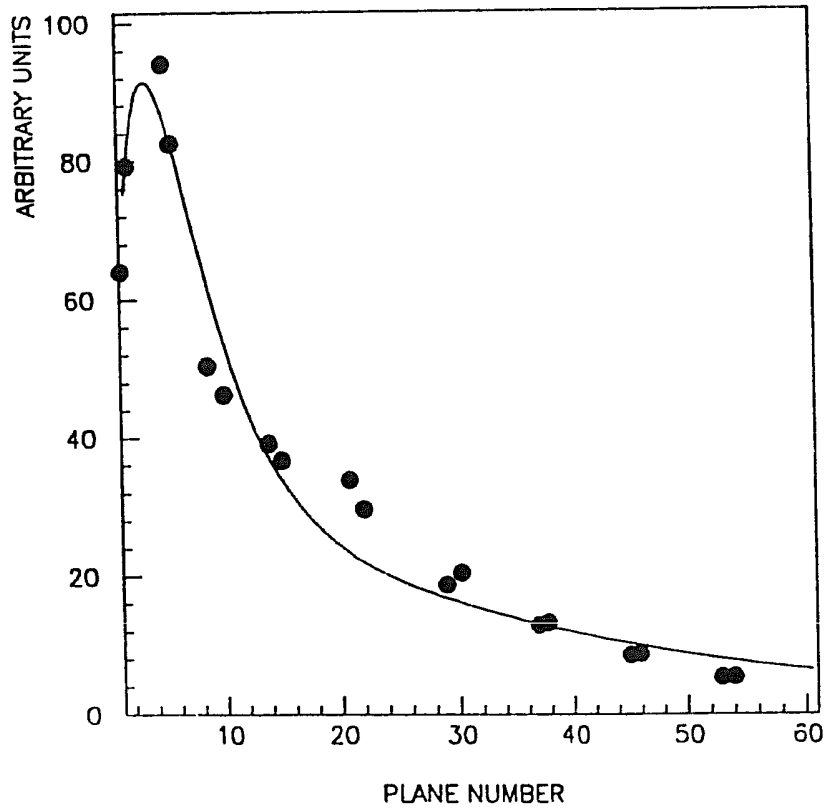

Fig. 10. Longitudinal distribution of hadronic showers with the interaction point in the front end of the HC. The curve is a result of the fit of data points to the function $C \exp (-A x)[1-$ $\exp (-B x)]+\exp \left(-A_{1} x\right)\left[1-\exp \left(-B_{1} x\right)\right]$, where $C=11.145$, $A=0.246, B=0.105, A_{1}=0.030$, and $B_{2}=1.216$. The $x$ is in planes of the HC. The thickness of one plane is $0.058 \lambda$.

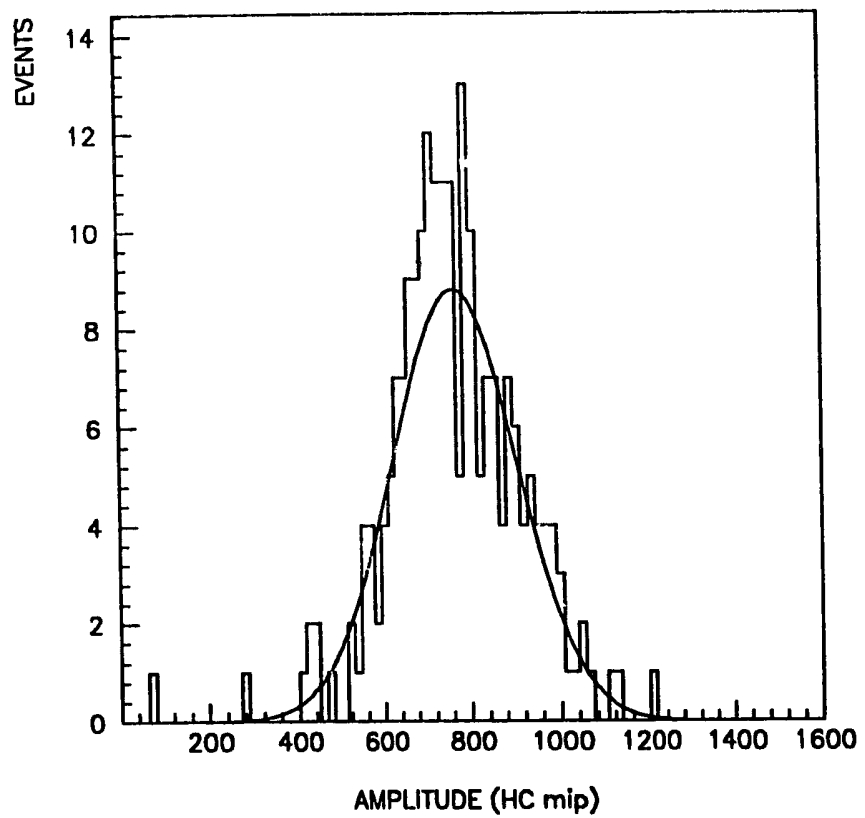

Fig. 11. The response to $20-\mathrm{GeV}$ pions originating in the front end of the HC. The curve is a Gaussian fit to the data. Mean response is $772.2 \mathrm{mip}, \sigma=18.1 \%$.

The distributions corresponding to the responses of the $\mathrm{HC}$ to $20 \mathrm{GeV}$ pions impinging on the HC-BGO setup are shown in figs. 12 to 14 . These include all events without restriction as to the shower starting point. As will be seen in the subsequent analysis the shape of these distributions arises from an event-to-event fluctuation (shrinkage and extension) of the shower in the longitudinal direction.

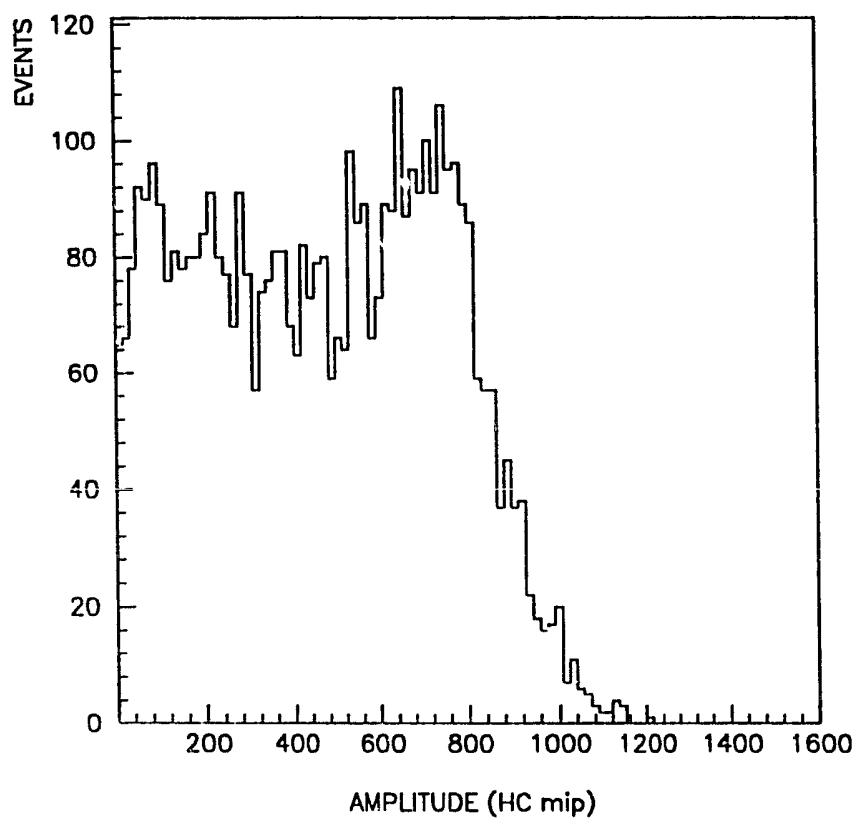

Fig. 12. The response to $20-\mathrm{GeV}$ pions of the $\mathrm{HC}$ situated behind the BGO matrix. 


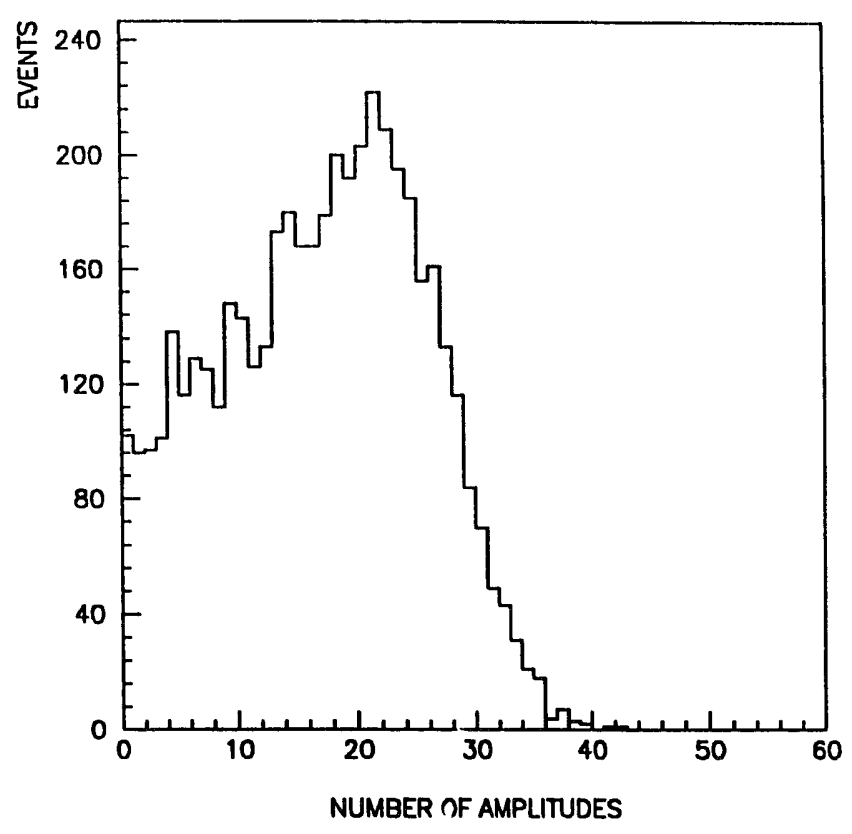

Fig. 13. Number of amplitudes $>6 \mathrm{mip}$ in $20-\mathrm{GeV}$ pion showers in the HC situated behind the BGO matrix.

\section{Parameters of the model}

As the large amplitudes give almost the totality of the signal (fig. 3), the parameters describing their spatial distribution are known with good accuracy from the data.

The signals with small amplitudes ( $<2 \mathrm{mip}$ ) contribute about $5 \%$ to the total signal. Because of their small contribution and small value the parameters connected with these amplitudes are determined from the data

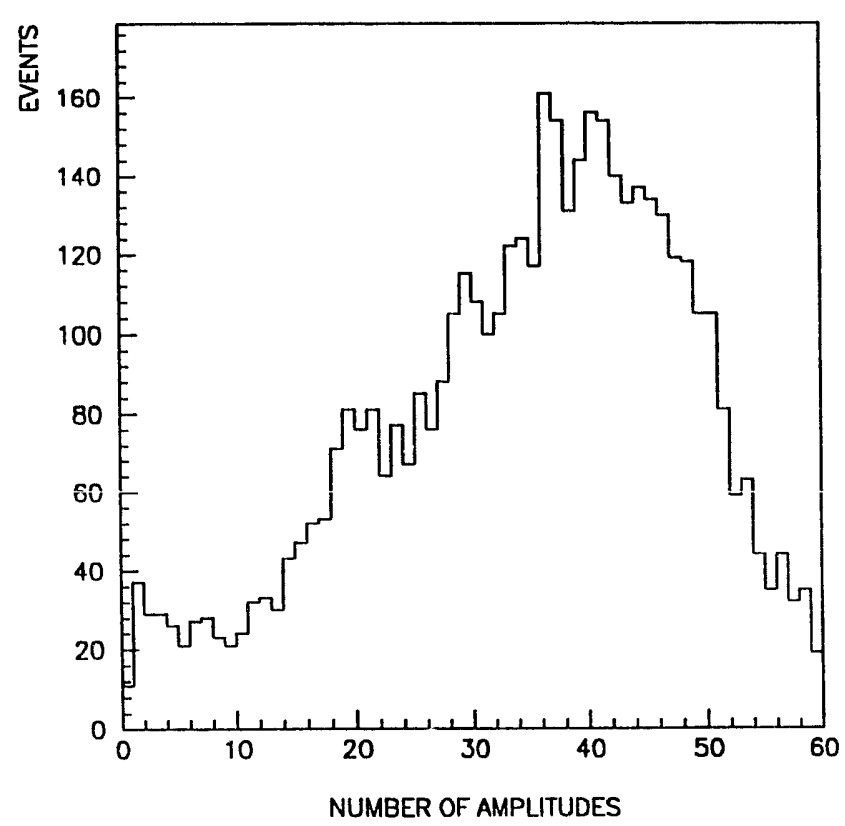

Fig. 14. Number of amplitudes $>6 \mathrm{mip}$ in $20-\mathrm{GeV}$ pion showers in the $\mathrm{HC}$ situated behind the BGO matrix.

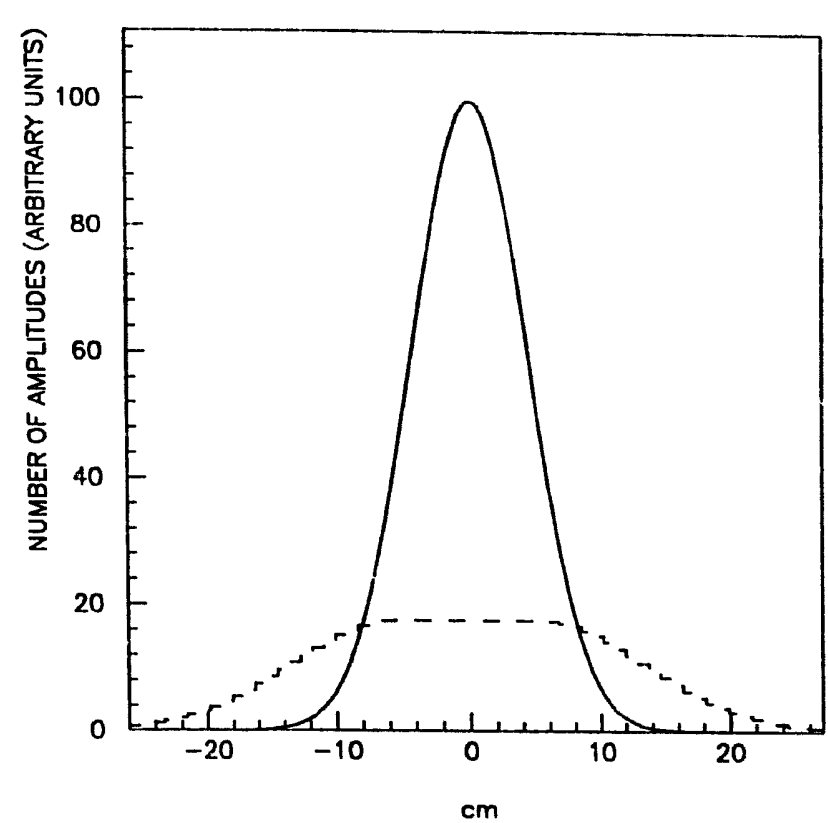

Fig. 15. Transverse distributions of large (solid line) and small (dashed line) signals used in simulation.

with less accuracy than the corresponding values for large signals.

The parametrized transverse distributions of different signals are shown in fig. 15. The large signals are described by a Gaussian with a standard deviation of 42

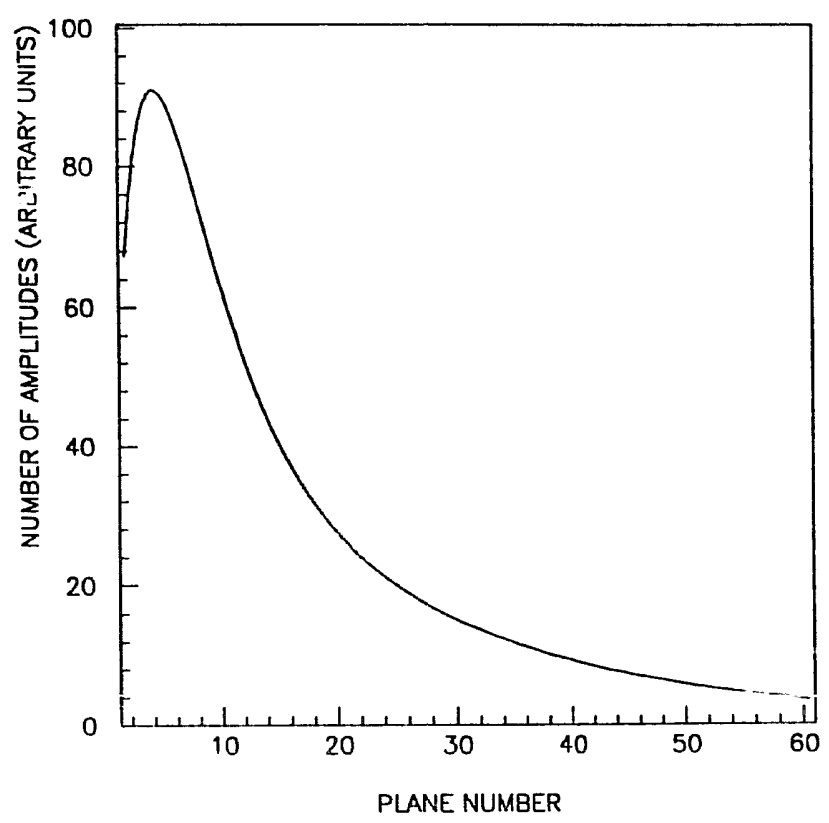

Fig. 16. Longitudinal $(20-\mathrm{GeV})$ shower distribution ased in simulation. This is given by the formula: $C \exp (-A x)[1-$ $\exp (-B x)]+\exp \left(-A_{1} x\right)\left[1-\exp \left(-B_{1} x\right)\right]$, where $C=5.56, A$ $=0.2, B=0.1 . A_{1}=0.045, B_{2}=0.7$. The $x$ is in planes of the HC. One plane is $0.058 \lambda$. The energy dependence is taken into account by varying $x$ as $x(E)=x / 0.5+0.163 \ln (E)$, where $E$ is in $\mathrm{GeV}$, and making the corresponding normalization. 
$\mathrm{mm}$. The distribution of small signals has a flat top of $100 \mathrm{~mm}$ and falls down as a Gaussian with a standard deviation of $42 \mathrm{~mm}$.

The longitudinal shape of the shower with the fixed starting rioint (fig. 16) is obtained from the measured one (fig. 10) through deconvolution. This procedure takes into account the fact that the measured shower is a superposition of showers originating in a $0.1 \lambda-0.15 \lambda$ deep region of the $\mathrm{HC}$.

The total response is a product of the number and the average amplitude of individual signals. However, for the given shape of the hadronic shower and readout-cell structure (fig. 2), using amplitude spectra (fig. 6) and the measured number of amplitudes above a given threshold (figs. 4 and 5), one can obtain the actual number and the average value of these signals. The number of small signals with an average amplitude of $1.6 \mathrm{mip}$ is $1.8 \mathrm{per} \mathrm{GeV}$. The corresponding values for large signals are $10.4 \mathrm{mip}$ and $3.6 \mathrm{per} \mathrm{GeV}$.

The number of both large and small amplitudes is supposed to be a linear function of the hadron energy. The spectra of amplitudes are described by exponential functions.

The scale parameter, which determines the event-toevent variation of the longitudinal shape of the shower, is obtained by fitting the distributions of simulated responses of the HC-BGO setup to data (fig. 12).

\section{Simulation of hadronic showers}

The basic element of simulation is an average hadronic shower, which gives the distribution of the number of small and large signals in the sensitive voiume of the HC. The longitudinal shape of this shower (fig. 16) evolves logarithmically with the energy of the simulated hadron, whereas its transverse shape (fig. 15) is assumed to be energy-independent. The actual energy dependence of the longitudinal shape was obtained from the data taken at $4,8,13$, and $20 \mathrm{GeV}$.

As mentioned above, the distribution of the HC responses, especially for events with the hadronic shower starting in the BGO, is very sensitive to variations of the longitudinal shape of the shower. In fig. 17 the simulated distributions with different scale factors for the longitudinal shower shape are presented. Comparing with data (fig. 2) one can conclude that no single factor can describe the experimental result. The best correspondence between the simulated and the measured resronse (fig. 18) is achieved when the longitudinal shape of each individual shower is corrected by a scale factor distributed as shown in fig. 19. Thus, the longitudinal scale of each simulated shower is multiplied by a factor randomly selected from the scale factor distribution.

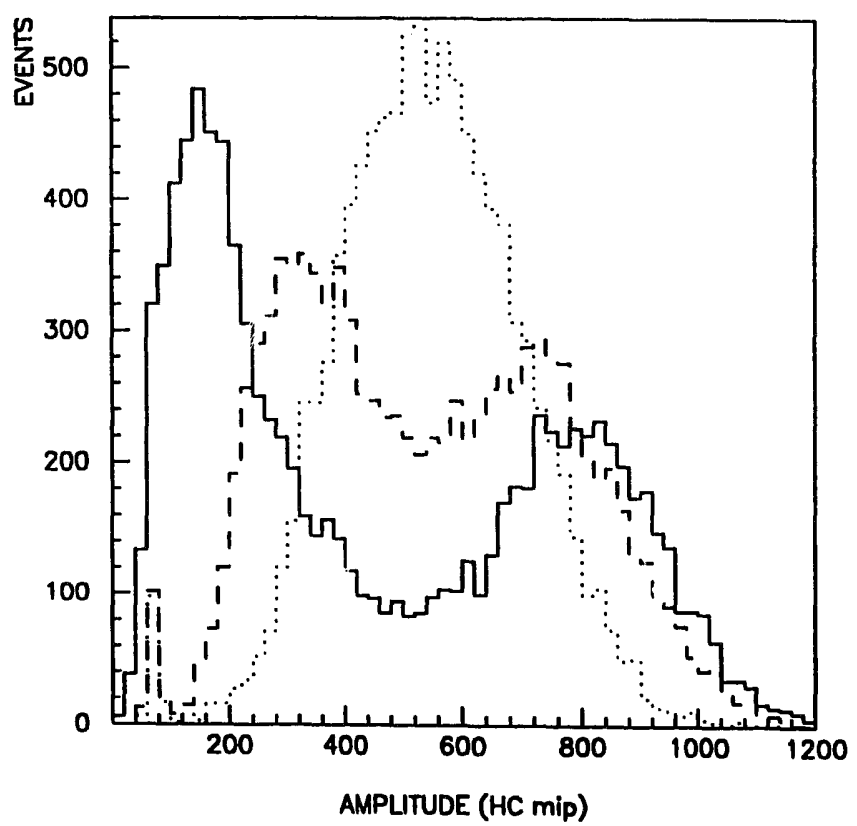

Fig. 17. Simulated response to $20-\mathrm{GeV}$ pions of the $\mathrm{HC}$ situated behind the BGO matrix. A solid line means scale factor $=0.5$, a dashed line means scale factor $=1.0$, and a dotted line means scale factor $=2.0$. The number of events is the same for all data sets.

For every simulated event, firstly the average number of small and large signal "hits" in each chamber plane is determined from the corresponding average shower. The number of "hits" obtained in the first step of simulation is considered as an average of a Poisson distribution. The actual number retained for further simulation of signals in the given readout cell is ran-

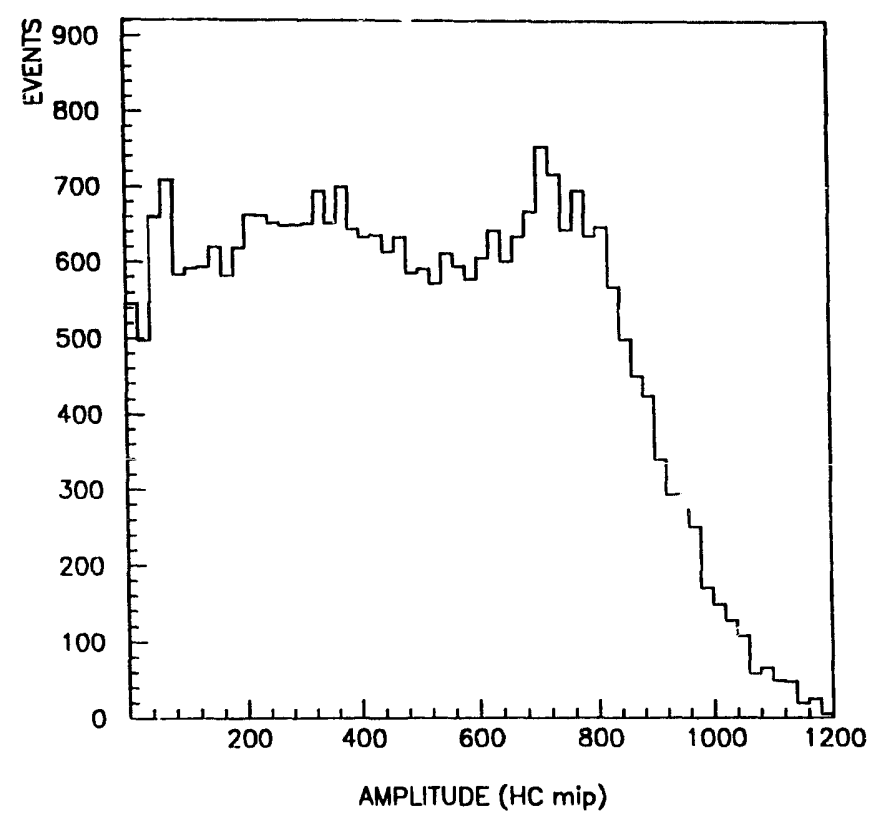

Fig. 18. Simulated response to $20-\mathrm{GeV}$ pions of the $\mathrm{HC}$ situated behind the BGO matrix. 


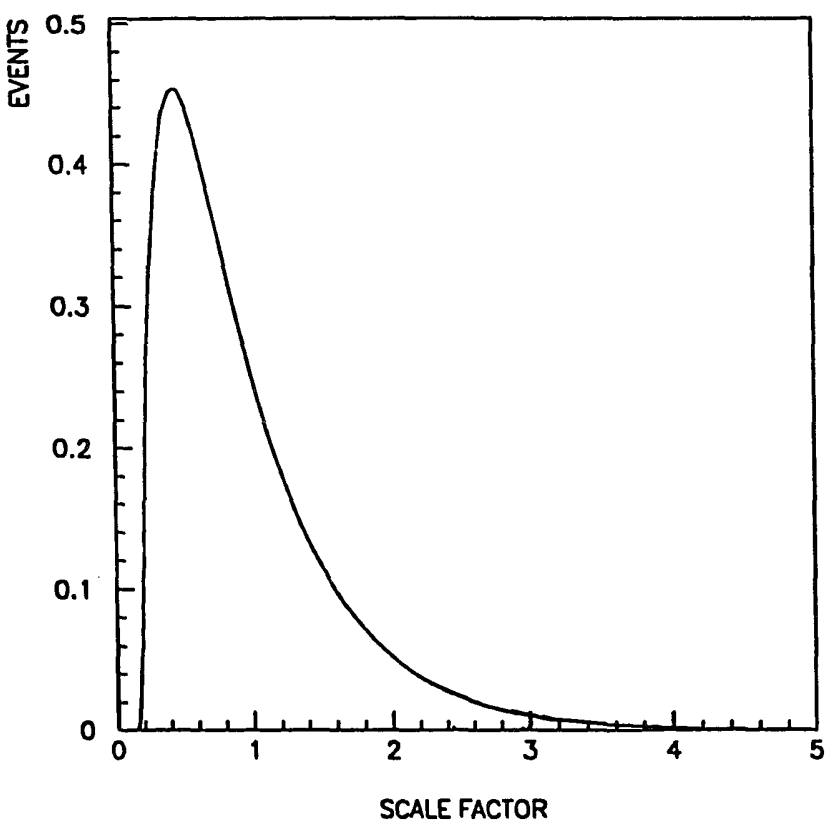

Fig. 19. Scale factor probability function.

domly selected from the Poisson distribution with the given average.

The spectra of signals with small and large amplitudes are assumed to be exponential and independent of the hidron energy. The amplitude of each individual signal is randomly selected from the corresponding spectra.

In the simulation of hadronic showers the starting point is distributed in the longitudinal direction in accordance with the nuclear interaction length.

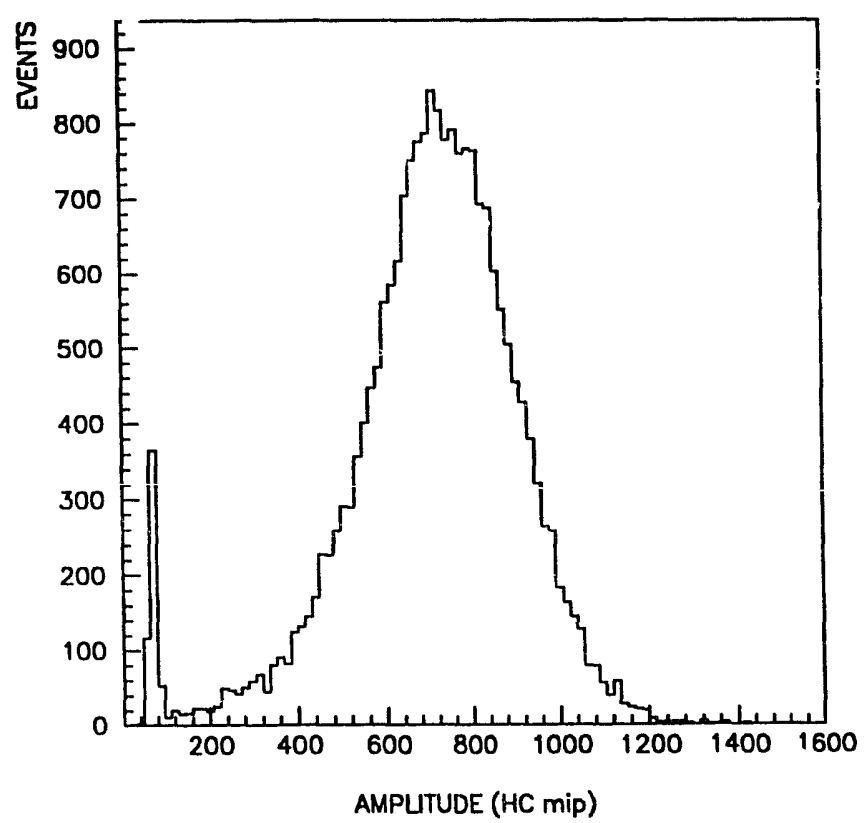

Fig. 20. Simulated $\mathrm{HC}$ response to $20-\mathrm{GeV}$ pions noninteracting in the BGO.

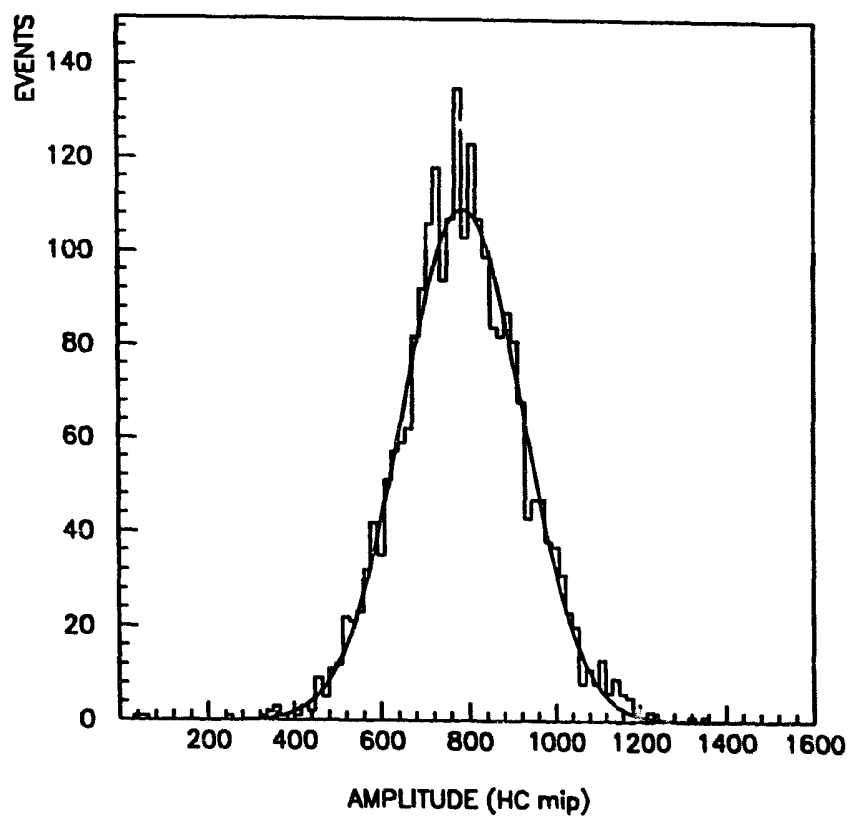

Fig. 21. Simulated response to $20-\mathrm{GeV}$ pions originating in the front end of the HC. The curve is a Gaussian fit to the data. Mean response $=787.3 \mathrm{mip}, \sigma=17.3 \%$.

Finally, the signals simulated in different parts of the HC volume are summed according to the existing readout pattern (fig. 2).

The result of the simulation of the $\mathrm{HC}$ response to $20 \mathrm{GeV}$ pions noninteracting in the BGO is presented in fig. 20. Similarly to the data, the asymmetry in the total response vanishes when the shower starting point is confined to the front end of the HC. The rms of

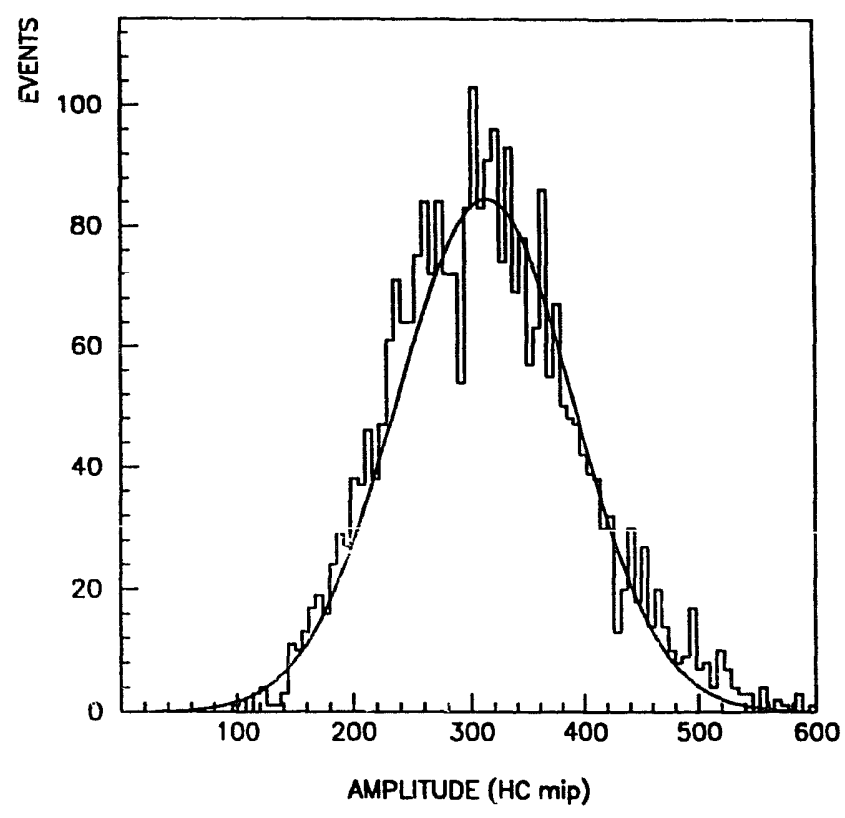

Fig. 22. Simulated response to $8-\mathrm{GeV}$ pions originating in the front end of the HC. The curve is a Gaussian fit to the data. Mean response $=313.4 \mathrm{mip}, \sigma=24.3 \%$. 


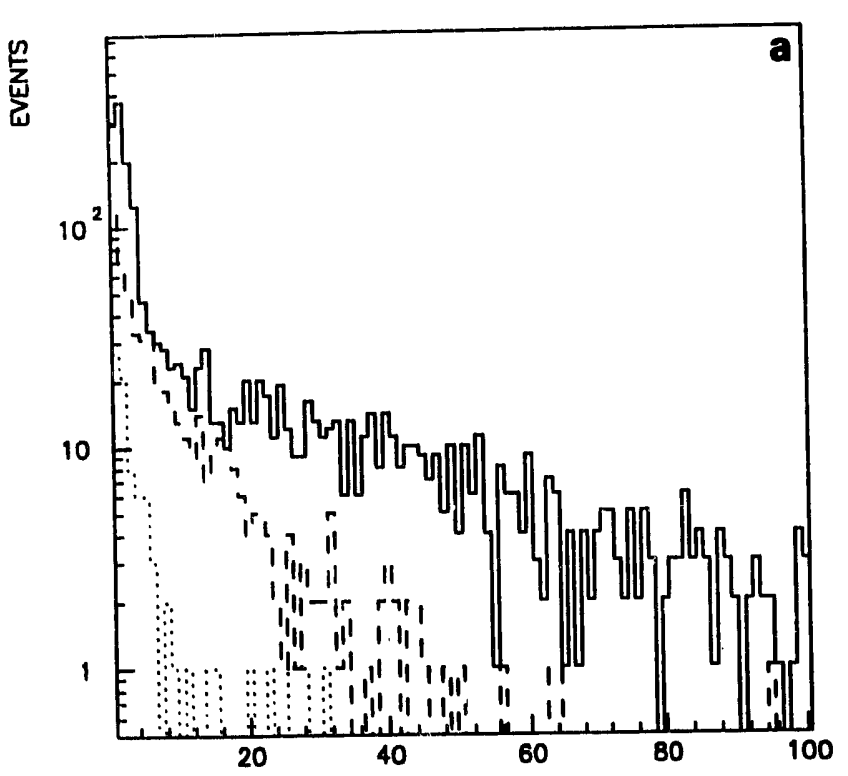

AMPLITUDE (HC mip)

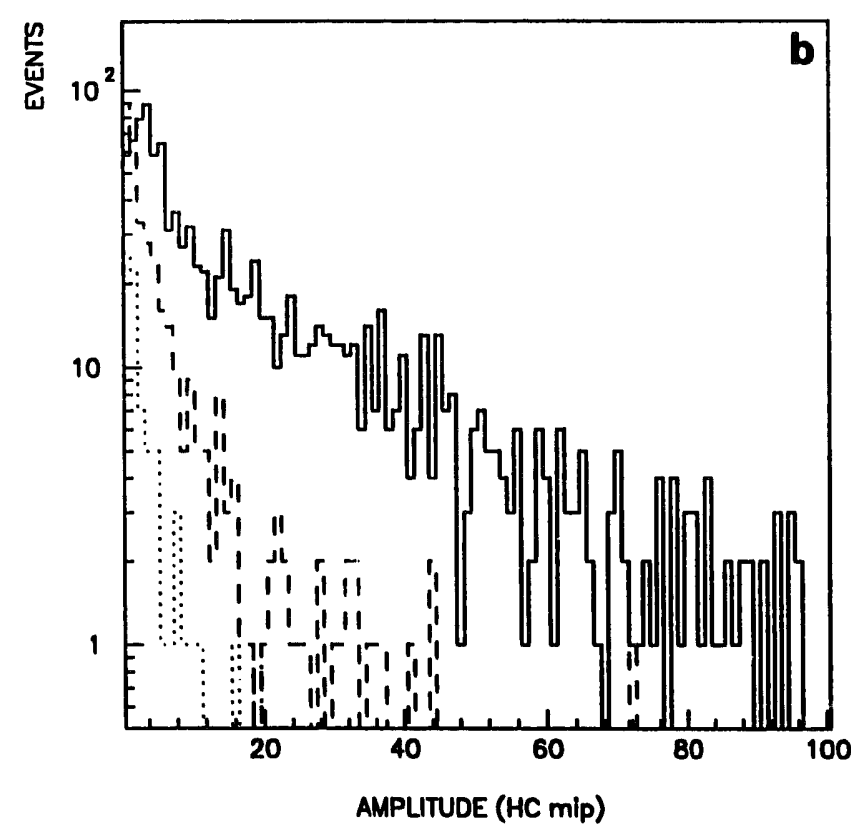

Fig. 23. Simulated amplitude spectra in different cells. A solid line means cell number 5 (central), a dast:ed line means cell number 3 (intermediate), and a dotted line means cell number 1 (peripheral); (a) layer number 3 (two chambers); (b) layer number 8 (four chambers).

distributions of simulated well-contained events (figs. 21 and 22) is in agreement with data [1]. The simulation not only describes well the overall characteristics of the HC response but also gives a fairly good description of amplitude spectra of individual readout cells (fig. 23).

The distribution of $\mathrm{fg} .12$ reflects the repartition of the hadronic shower between the BGO and the HC. This is also presented as a scatte: plot in the HC-BGO
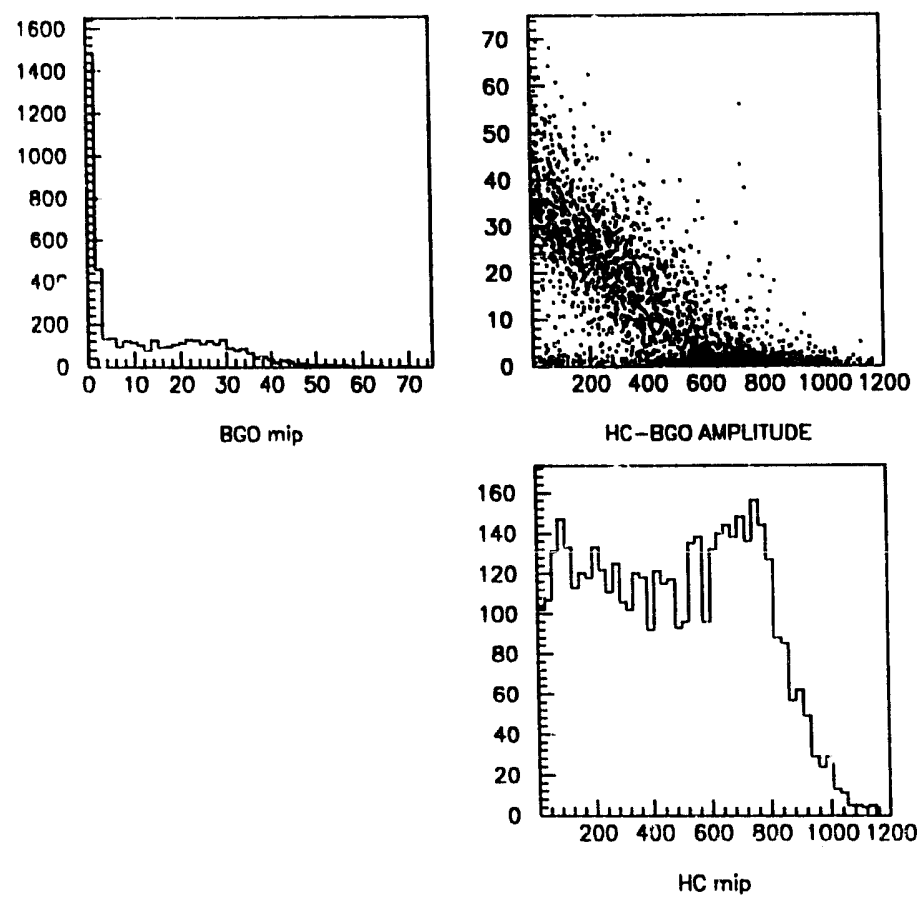

Fig. 24. Scatter plot projectiors if the HC-BGO response; 20-GeV data. signal plane in figs. 24 and 25 . In this plot the BGO signal is measured in mip in the BGO. This is equal to $267 \mathrm{MeV}$.

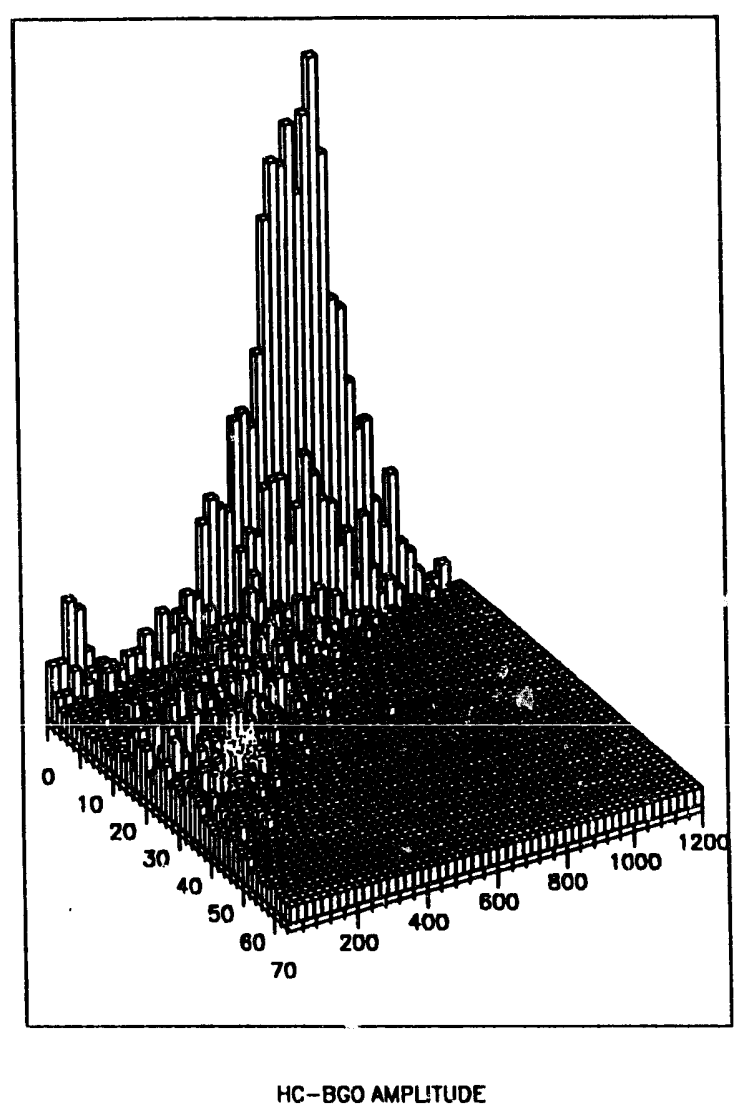

Fig. 25. Scatter plot of the HC-BGO response; $20-\mathrm{GeV}$ data. 

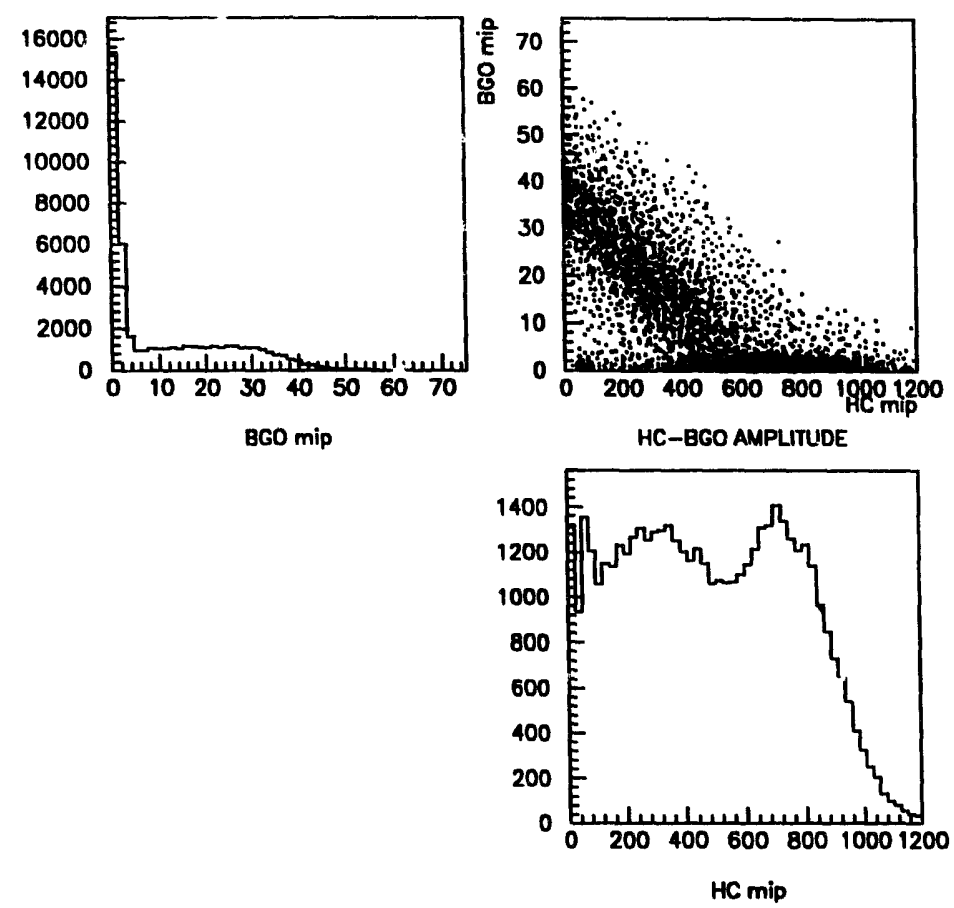

Fig. 26. Scatter plot jrojections of the HC-BGO response; 20-GeV simulation.

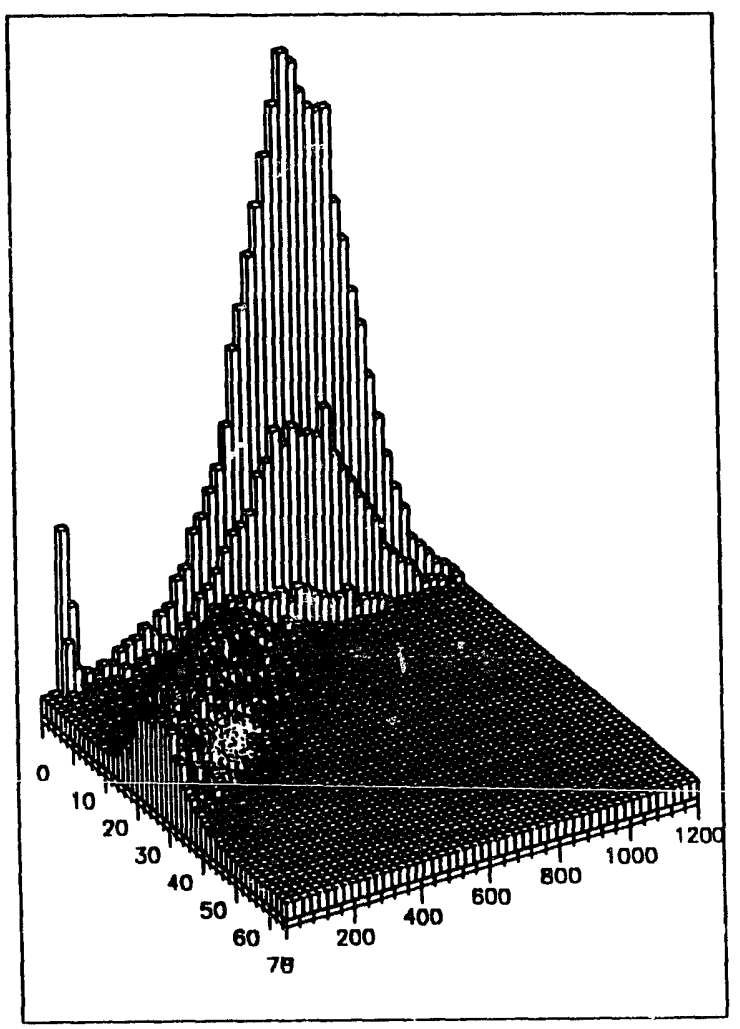

HC-BGO AMPLITUDE

Fig. 27. Scatter plot of the HC-BGO respunse; $20-\mathrm{GeV}$ simulation.
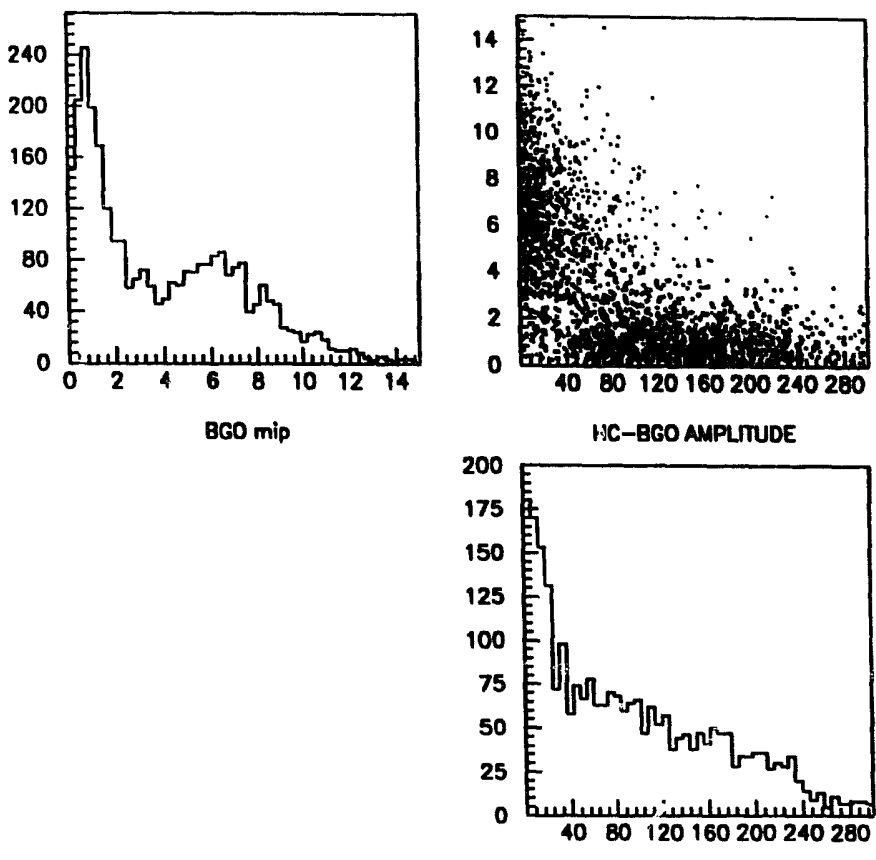

HC mip

Fig. 28. Scatter plot projections of the HC-BGO response; 4-GeV data.

A similar scatter plot for simulated hadronic showers is presented in figs. 26 and 27 . To obtain the signal in the BGO the simulation is performed as if the whole setup consisted of two gas-sampling calorinieters: one $0.93 \lambda$ (corresponding to the thickness of the $B G O$ ) and the other $3.36 \lambda$ thick (corresponding to the HC), sep-
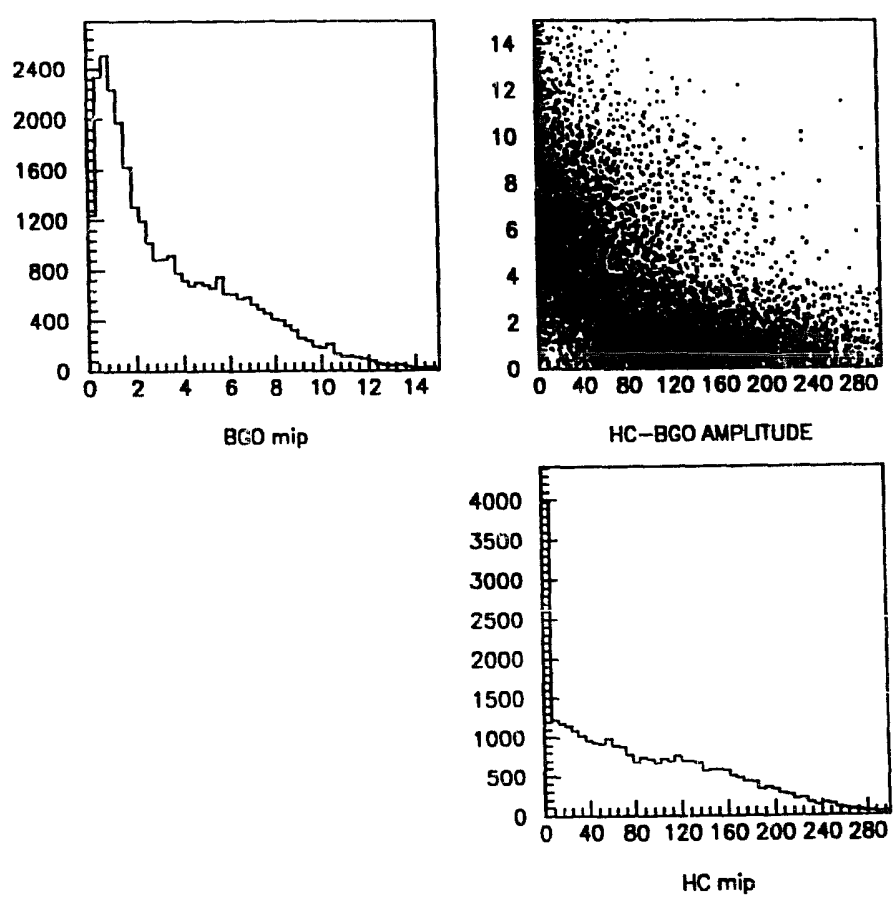

Fig. 29. Scatter plot projections of the HC-BGO response; 4-GeV simulation. 

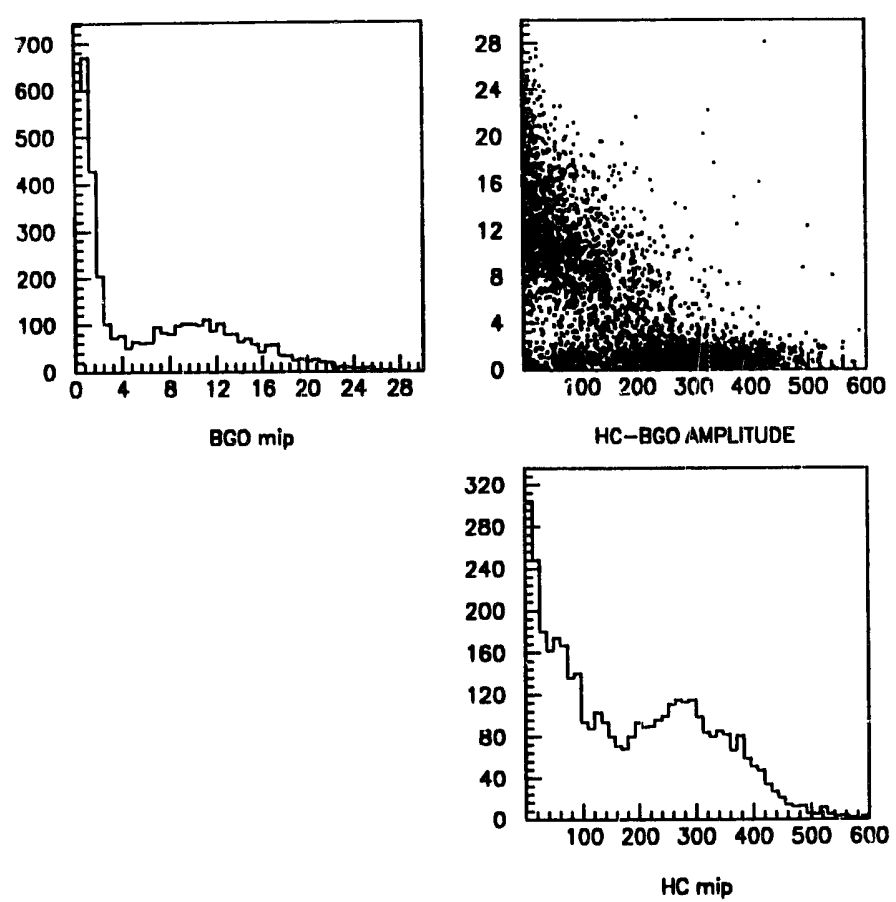

Fig. 30. Scatter plot projections of the HC-BGO response; 8-GeV data.
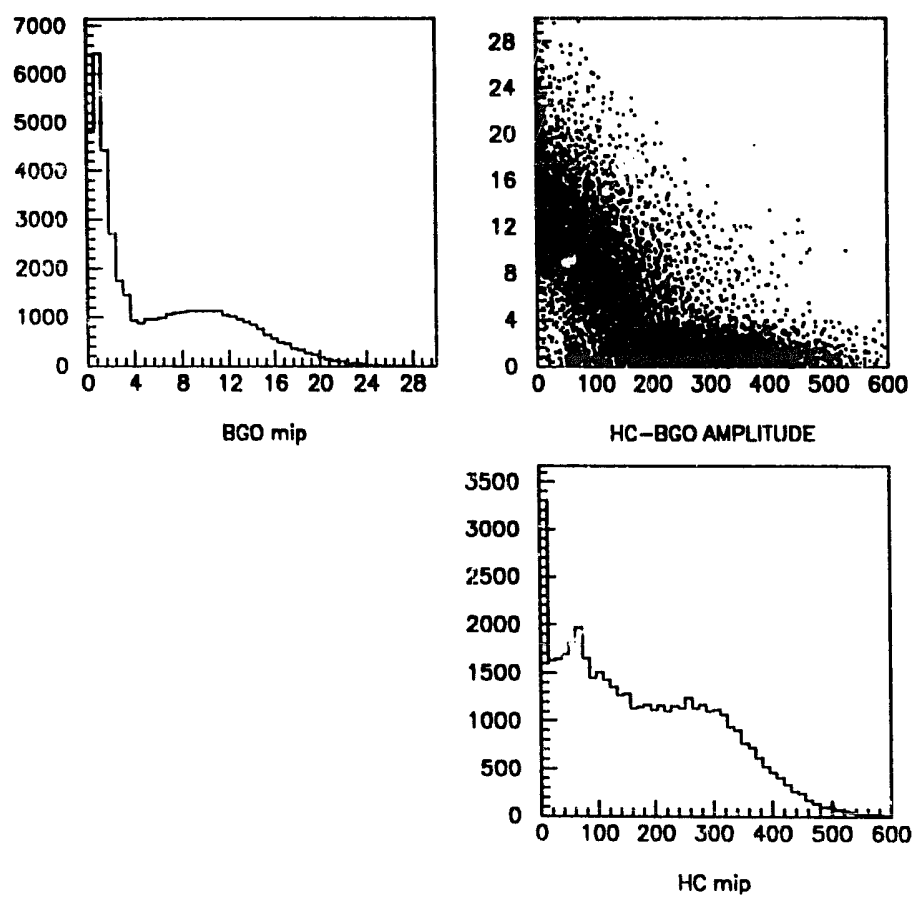

Fig. 31. Scatter plot projections of the HC-BGO response; 8-GeV simulation. arated by a $0.21 \lambda$ thick dead region (steel support ring). The simulated signal from the front part of the $\mathrm{HC}$ is called a "BGO" signal. The part of the shower in the wall between the BGO and the $\mathrm{HC}$ is considered as lost for detection.

It turns out that once the "BGO" signal scale is properly adjusted at one energy (namely $20 \mathrm{GeV}$ ), the simulated data show good agreement with the real data at other pion energies (figs. 28-31).

\section{Conclusions}

The data show that in a uranium gas-sampling calorimeter filled with a gas containing no hydrogen the detected hadronic shower is to a large extent contained in a narrow (rms $=42 \mathrm{~mm}$ ) region surrounding the initial particle direction. In the longitudinal direction the average shower has a narrow (about $0.7 \lambda$ at $20 \mathrm{GeV}$ or $15 X_{0}$ ( $X_{0}$ is the radiation length) FWHM) peak in the beginning. The peak contains about $75 \%$ of the total shower.

The calorimeter response is mainly dominated by a small number of large signals.

The Monte Carlo program, based on the parametrization of the test data, provides a tool for simulation of hadronic showers in the $\mathrm{L} 3$ detector. It reproduces the test results at different energies and angles of incidence of the pions.

\section{References}

[1] L3 Collaboration, The construction of the L3 experiment, to be published in Nucl. Instr. and Meth. A.

[2] J.A. Bakken et al., Nucl. Instr. and Meth. 228 (1985) 294.

[3] J.A. Bakken e؛ al., Nucl. Instr. and Meth. A254 (1987) 535.

[4] A Arefiev et al., Nucl. Instr. and Meth. A275 (1989) 71. 